\title{
CLASSICAL ANALOGUE OF WEAK VALUE IN STOCHASTIC PROCESS
}

\author{
H. TOMITA \\ Research Center of Quantum Computing, Kinki University \\ Higashi-Osaka, 577-8502, Japan \\ E-mail: tomita@alice.math.kindai.ac.jp
}

\begin{abstract}
One of the remarkable notions in the recent development of quantum physics is the weak value related to weak measurements. We emulate it as a two-time conditional expectation in a classical stochastic model. We use the well known symmetrized form of the master equation, which is formally equivalent to the wave equation in quantum mechanics apart from the fact that wave functions are always real. The origin of the unusual behaviors of the weak value such as the negative probability and the abnormal enhancement of some expectations becomes clearer in the present case, where the two-time conditional probability has no ambiguity of imaginary/complex values.

Keywords: Weak value, Stochastic process, Two-time conditional probability, Stochastic Ising model
\end{abstract}

\section{Introduction}

The weak value is a derived notion of the weak measurement proposed by Aharonov et al, ${ }^{1}$ which has brought a new understanding of quantum observations. The weak measurement ${ }^{2}$ means that it hardly disturbs the quantum superposed state when it is performed with large uncertainty. The reason of the strange nature of this quantum measurement is that the weak value is defined as an expectation over strongly restricted paths with the condition of the post-selected final state.

Suppose we have started from a pre-selected initial state at $t=t_{\mathrm{i}}$. If we measure some observable $Q$ at $t\left(>t_{\mathrm{i}}\right)$, the wave function $\Psi(t)$ collapses to one of the eigenstates of $Q$. And we can find only the probability distribution $|\Psi(t)|^{2}$, not the probability amplitude $\Psi(t)$, by repeating the measurement and adopting all of the observed data. If we discard the main data by restricting the paths to the post-selected ones only, we can expect to get some informations on the state $\Psi(t)$ before collapsing, because the 
post-selected paths are described with the same propagator as the forward evolution of the pre-selected paths by changing only the sign of the time $t$ according to the time-reversal symmetry of quantum mechanics.

The weak value of an observable $Q$ with a given initial state $|i\rangle$ at $t=t_{\mathrm{i}}$ and a final state $|f\rangle$ at $t=t_{\mathrm{f}}$ is defined by ${ }^{3}$

$$
\langle Q\rangle_{(\mathrm{f} ; \mathrm{i})}^{\mathrm{w}}=\frac{\left\langle f\left|\mathrm{e}^{-i\left(t_{\mathrm{f}}-t\right) H} Q \mathrm{e}^{-i\left(t-t_{\mathrm{i}}\right) H}\right| i\right\rangle}{\left\langle f\left|\mathrm{e}^{-i\left(t_{\mathrm{f}}-t_{\mathrm{i}}\right) H}\right| i\right\rangle},\left(t_{\mathrm{i}} \leq t \leq t_{\mathrm{f}}\right)
$$

where $H$ is the Hamiltonian and the unit $\hbar=1$ is used. This quantity is related to a weak measurement as follows: Let us introduce a meter to measure the observable $Q$ of the target system at $t=t_{0}$ by a weak interaction

$$
H_{\text {int }}(t)=g \delta\left(t-t_{0}\right) Q \otimes p,
$$

where $p$ is the momentum operator of the probe of the meter and $g$ is a small coupling constant. Suppose the initial state of the meter, $\varphi(x)$ in the coordinate representation of the probe position $x$ has a sufficiently broad uncertainty $\Delta$, i.e. a variance $\Delta^{2}$. It can be easily shown that for the restricted paths from $i$ to $f$, the meter state for $t>t_{0}$ is given by

$$
\begin{aligned}
& \left\langle f\left|\mathrm{e}^{-i\left(t_{\mathrm{f}}-t\right) H} \mathrm{e}^{-i g Q \otimes p} \mathrm{e}^{-i\left(t-t_{\mathrm{i}}\right) H}\right| i\right\rangle \varphi(x) \\
\simeq & \left\langle f\left|\mathrm{e}^{-i\left(t_{\mathrm{f}}-t_{\mathrm{i}}\right) H}\right| i\right\rangle \varphi\left(x-g\langle Q\rangle_{(\mathrm{f} ; \mathrm{i})}^{\mathrm{w}}\right),
\end{aligned}
$$

where $p=-i \partial / \partial x$ is used. Note that the weak value defined by Eq.(1) is complex in general. Then the shift of the expectation of the probe position $x$ is given by the real part of the weak value, $g \operatorname{Re}\left[\langle Q\rangle_{(\mathrm{f} ; \mathrm{i})}^{\mathrm{w}}\right]$, while the shift of the expectation of the momentum $p$ is found to be equal to the imaginary part, $\left(g / 2 \Delta^{2}\right) \operatorname{Im}\left[\langle Q\rangle_{(\mathrm{f} ; \mathrm{i})}^{\mathrm{w}}\right]$ by using a Fourier transformation.

An early interpretation of the quantity defined by Eq.(1) is the probability amplitude 4 which yields a pre- and post-selected, two-time conditional probability (TTCP). When it is applied to a projection operator $|q\rangle\langle q|$ onto one of the eigenstates of an observable $Q$, one finds

$$
\left|\langle\mid q\rangle\langle q \mid\rangle_{(\mathrm{f} ; \mathrm{i})}^{\mathrm{w}}\right|^{2}=\left|\frac{\left\langle f\left|\mathrm{e}^{-i\left(t_{\mathrm{f}}-t\right) H}\right| q\right\rangle\left\langle q\left|\mathrm{e}^{-i\left(t-t_{\mathrm{i}}\right) H}\right| i\right\rangle}{\left\langle f\left|\mathrm{e}^{-i\left(t_{\mathrm{f}}-t_{\mathrm{i}}\right) H}\right| i\right\rangle}\right|^{2}=\frac{P(f \mid q) P(q \mid i)}{P(f \mid i)} .
$$

The last expression is to be shown equal to $P(q \mid f \cap i)$ in Sec.3 by using Bayes identities. (See the footnote c in Sec.3.) Here $P(* \mid C)$ is the standard notation for a conditional probability with a condition $C$ (or a transition probability from the state $C$ to $*$ ), e.g.

$$
P(f \mid q)=\left|\left\langle f\left|\mathrm{e}^{-i\left(t_{\mathrm{f}}-t\right) H}\right| q\right\rangle\right|^{2}, \text { etc. }
$$


Another interpretation, somewhat formal one, is the TTCP itself. ${ }^{3,5}$ Let us rewrite the usual quantum expectation of $Q(t)=\mathrm{e}^{i\left(t-t_{\mathrm{i}}\right) H} Q \mathrm{e}^{-i\left(t-t_{\mathrm{i}}\right) H}$ with respect to a state $|i\rangle$ in the following identical form,

$$
\begin{aligned}
\langle i|Q(t)| i\rangle & =\sum_{f}\left\langle i\left|\mathrm{e}^{i\left(t_{\mathrm{f}}-t_{\mathrm{i}}\right) H}\right| f\right\rangle\left\langle f\left|\mathrm{e}^{-i\left(t_{\mathrm{f}}-t\right) H} Q \mathrm{e}^{-i\left(t-t_{\mathrm{i}}\right) H}\right| i\right\rangle \\
& =\sum_{f}\langle Q\rangle_{(\mathrm{f} ; \mathrm{i})}^{\mathrm{w}}\left|\left\langle f\left|\mathrm{e}^{-i\left(t_{\mathrm{f}}-t_{\mathrm{i}}\right) H}\right| i\right\rangle\right|^{2},
\end{aligned}
$$

where the last factor of Eq.(4) is $P(f \mid i)$. This may yield an interpretation of the weak value as a complex, raw stochastic variable. Nevertheless, if it is applied to $|q\rangle\langle q|$ again, it reads as a conditional probability equation,

$$
P(q \mid i)=\left|\left\langle q\left|\mathrm{e}^{-i\left(t-t_{\mathrm{i}}\right) H}\right| i\right\rangle\right|^{2}=\sum_{f}\langle\mid q\rangle\left\langle\left. q\right|_{(\mathrm{f} ; \mathrm{i})} ^{\mathrm{w}} P(f \mid i) .\right.
$$

In addition, we have a sum-rule,

$$
\sum_{q}\langle\mid q\rangle\langle q \mid\rangle_{(\mathrm{f} ; \mathrm{i})}^{\mathrm{w}}=1
$$

because of the completeness, $\sum_{q}|q\rangle\langle q|=I$ (= identity). Therefore, if we remind a type of the Bayes statistics relations, ${ }^{\text {a }}$

$$
P(q \mid i)=\sum_{f} P(f \cap q \mid i)=\sum_{f} P(q \mid f \cap i) P(f \mid i),
$$

the weak value of the projection operator $|q\rangle\langle q|$, though it may be complex, can be interpreted formally as a TTCP itself with a couple of pre- and postselections, $i$ and $f$. Further, the weak value of an operator $Q=\sum_{q} q|q\rangle\langle q|$, or $A=\sum_{q} a(q)|q\rangle\langle q|$ in general, can be interpreted as the two-time conditional expectations (TTCE) of them with respect to this virtual TTCP.

Because of this rather fictitious interpretation, the virtual conditional probability happens to be negative ${ }^{6}$ and it causes an abnormal enhancement of the weak value of some observables greater than their inherent norms. ${ }^{5}$ These strange behaviors are closely related. That is, at least if a probability set $\{P(q)\}$ is real, it can be expected that we have a partial sum satisfying

$$
\sum_{P(q) \geq 0} P(q)=1-\sum_{P(q)<0} P(q)>1,
$$

whenever there exists a negative part. This is the essential reason of a possibility of the unusual enhancement of some expectations. ${ }^{6,7}$

$\overline{\text { a } P(f \cap q \mid i)=P(q \cap f \cap i) / P(i)=} P(q \mid f \cap i) P(f \cap i) / P(i)=P(q \mid f \cap i) P(f \mid i)$

For the Bayes identity, see the equation $(*)$ in the footnote c in Sec.3. 
The purpose of the present work is to emulate these strange behaviors clearer by using a classical stochastic model, in which we can avoid the ambiguity of the complex probability in the above quantum problem. ${ }^{8} \mathrm{We}$ survey a conventional transformation of the stochastic master equation to a self-adjoint form in the following section. A good analogy with the quantum mechanics is found by applying it to the TTCP. This is shown in Sec.3. An example of the stochastic Ising model which shows an abnormal enhancement of the expectations of some quantities with respect to TTCP is given in Sec.4. In Sec.5 we discuss an extension of TTCP to a density matrix form to complete the analogy with the quantum mechanics. The last section is devoted to brief summary and discussions.

\section{Self-adjoint form of stochastic master equation}

First let us survey the well-known transformation ${ }^{9}$ to a self-adjoint form of the stochastic master equation.

Let $\boldsymbol{x}$ be a set of stochastic variable(s) described by a time-dependent conditional probability, $P\left(\boldsymbol{x}, t \mid \boldsymbol{x}_{\mathrm{i}}, t_{\mathrm{i}}\right)$ for $t \geq t_{\mathrm{i}}$, which obeys the following stationary, Markovian master equation, i.e. the Chapman-Kolmogorov forward equation,

$$
\begin{aligned}
\frac{\partial}{\partial t} P\left(\boldsymbol{x}, t \mid \boldsymbol{x}_{\mathrm{i}}, t_{\mathrm{i}}\right)= & -\sum_{\boldsymbol{x}^{\prime}} W\left(\boldsymbol{x} \rightarrow \boldsymbol{x}^{\prime}\right) P\left(\boldsymbol{x}, t \mid \boldsymbol{x}_{\mathrm{i}}, t_{\mathrm{i}}\right) \\
& +\sum_{\boldsymbol{x}^{\prime}} W\left(\boldsymbol{x}^{\prime} \rightarrow \boldsymbol{x}\right) P\left(\boldsymbol{x}^{\prime}, t \mid \boldsymbol{x}_{\mathrm{i}}, t_{\mathrm{i}}\right) \\
= & -\sum_{\boldsymbol{x}^{\prime}} L\left(\boldsymbol{x}, \boldsymbol{x}^{\prime}\right) P\left(\boldsymbol{x}^{\prime}, t \mid \boldsymbol{x}_{\mathrm{i}}, t_{\mathrm{i}}\right)
\end{aligned}
$$

where

$$
L\left(\boldsymbol{x}, \boldsymbol{x}^{\prime}\right)=\delta\left(\boldsymbol{x}-\boldsymbol{x}^{\prime}\right) \sum_{\boldsymbol{x}^{\prime \prime}} W\left(\boldsymbol{x} \rightarrow \boldsymbol{x}^{\prime \prime}\right)-W\left(\boldsymbol{x}^{\prime} \rightarrow \boldsymbol{x}\right) .
$$

The matrix $L$ has an eigenvalue $\lambda_{0}=0$ corresponding to the steady state,

$$
P_{0}(\boldsymbol{x})=\lim _{t-t_{\mathrm{i}} \rightarrow \infty} P\left(\boldsymbol{x}, t \mid \boldsymbol{x}_{\mathrm{i}}, t_{\mathrm{i}}\right) .
$$

Let us introduce a wave function related to this forward conditional probability by

$$
\psi\left(\boldsymbol{x}, t \mid \boldsymbol{x}_{\mathrm{i}}, t_{\mathrm{i}}\right)=\phi_{0}(\boldsymbol{x})^{-1} P\left(\boldsymbol{x}, t \mid \boldsymbol{x}_{\mathrm{i}}, t_{\mathrm{i}}\right), \quad\left(t \geq t_{\mathrm{i}}\right)
$$


where $\phi_{0}(\boldsymbol{x})=P_{0}(\boldsymbol{x})^{1 / 2}$. This function $\psi$ obeys the forward wave equation,

$$
\frac{\partial}{\partial t} \psi(\boldsymbol{x}, t)=-\sum_{\boldsymbol{x}^{\prime}} H\left(\boldsymbol{x}, \boldsymbol{x}^{\prime}\right) \psi\left(\boldsymbol{x}^{\prime}, t\right),
$$

where $H$ is defined by

$$
H\left(\boldsymbol{x}, \boldsymbol{x}^{\prime}\right)=\phi_{0}(\boldsymbol{x})^{-1} L\left(\boldsymbol{x}, \boldsymbol{x}^{\prime}\right) \phi_{0}\left(\boldsymbol{x}^{\prime}\right) .
$$

For the time being the initial condition $\left(\boldsymbol{x}_{\mathrm{i}}, t_{\mathrm{i}}\right)$ in $\psi$ is abbreviated. The function $\phi_{0}(\boldsymbol{x})$ is an eigenfunction of Eq.(9) for $\lambda_{0}=0$.

The merit of this transformation is that the eigenvalue problem of a given master equation is simplified, if the matrix $H$ is symmetric, i.e.

$$
H\left(\boldsymbol{x}, \boldsymbol{x}^{\prime}\right)=H\left(\boldsymbol{x}^{\prime}, \boldsymbol{x}\right) .
$$

This situation is widely expected when the detailed balance condition, i.e. the time-reversal symmetry, ${ }^{10}$

$$
P_{0}(\boldsymbol{x}) W\left(\boldsymbol{x} \rightarrow \boldsymbol{x}^{\prime}\right)=P_{0}\left(\boldsymbol{x}^{\prime}\right) W\left(\boldsymbol{x}^{\prime} \rightarrow \boldsymbol{x}\right),
$$

or equivalently,

$$
L\left(\boldsymbol{x}, \boldsymbol{x}^{\prime}\right) P_{0}\left(\boldsymbol{x}^{\prime}\right)=L\left(\boldsymbol{x}^{\prime}, \boldsymbol{x}\right) P_{0}(\boldsymbol{x}),
$$

is satisfied. ${ }^{\mathrm{b}}$ In this case the eigenvalues of $H$ are all real, and non-negative, if the steady state is stable. Therefore, $\phi_{0}(\boldsymbol{x})$ is the ground state.

A useful example is the Fokker-Planck equation for a single, continuous stochastic variable $x$,

$$
\frac{\partial}{\partial t} P(x, t)=-\mathcal{L}[x] P(x, t), \mathcal{L}[x]=-\frac{\partial}{\partial x}\left(F^{\prime}(x)+\frac{\epsilon}{2} \frac{\partial}{\partial x}\right),
$$

which describes a one-dimensional Brownian motion in a potential $F(x)$ with a small diffusion constant $\epsilon$. By using its steady state solutions,

$$
P_{0}(x) \propto \exp [-2 F(x) / \epsilon] \text { and } \phi_{0}(x) \propto \exp [-F(x) / \epsilon],
$$

we find the continuous variable version of the above formulations,

$$
\mathcal{H}[x]=\frac{1}{\epsilon}\left[-\frac{\epsilon^{2}}{2} \frac{\partial^{2}}{\partial x^{2}}+V(x)\right], \quad V(x)=\frac{1}{2}\left[F^{\prime}(x)^{2}-\epsilon F^{\prime \prime}(x)\right] .
$$

\footnotetext{
bIt should be noted that the time reversal symmetry is assumed in this form of the probability flow and not on the transition probability itself, the latter being satisfied in quantum mechanics. This is the reason why we need the above transformation to obtain a self-adjoint formulation like quantum mechanics.
} 

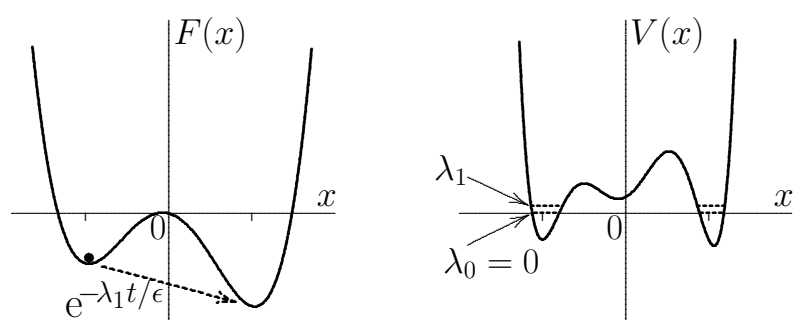

Fig. 1. Stochastic decay process of the metastable state.

Thus the Fokker-Planck equation is transformed into a self-adjoint form of an imaginary-time Schrödinger equation,

$$
-\epsilon \frac{\partial}{\partial t} \psi(x, t)=\left[-\frac{\epsilon^{2}}{2} \frac{\partial^{2}}{\partial x^{2}}+V(x)\right] \psi(x, t),
$$

and its eigenvalue problem results in a familiar one of the quantum mechanics.

Figure.1 shows an early application ${ }^{11}$ to the so-called Kramers escape problem. The stochastic decay (or escape) rate of the metastable state in a double-well potential $F(x)$ is given by the first excited eigenvalue $\lambda_{1}$ of the corresponding Schrödinger potential $V(x)$. The first excited state is almost degenerate with the ground state for a small diffusion constant $\epsilon$.

\section{Two-time conditional probability}

So far the quantum mechanical reformulation merely helps us to simplify the eigenvalue problem of a given master equation. None of remarkable quantum mechanical phenomena appears, until we are concerned with the TTCP,

$$
P\left(\boldsymbol{x}, t \mid \boldsymbol{x}_{\mathrm{f}}, t_{\mathrm{f}} ; \boldsymbol{x}_{\mathrm{i}}, t_{\mathrm{i}}\right), \quad t_{\mathrm{i}} \leq t \leq t_{\mathrm{f}} . \quad(; \text { denoting 'and', or } \cap)
$$

By using the Markovian property and the well-known relation between joint and conditional probabilities repeatedly, ${ }^{\mathrm{c}}$ the TTCP can be written in the

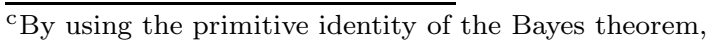

$$
P(A \mid B) P(B)=P(B \mid A) P(A)=P(A \cap B),
$$

we find in abbreviated notations,

$$
P(x \mid f \cap i)=\frac{P(f \cap x \cap i)}{P(f \cap i)}=\frac{P(f \mid x \cap i) P(x \cap i)}{P(f \cap i)}=\frac{P(f \mid x) P(x \mid i) P(i)}{P(f \cap i)},
$$


following form with a pair of wave functions as

$$
P\left(\boldsymbol{x}, t \mid \boldsymbol{x}_{\mathrm{f}}, t_{\mathrm{f}} ; \boldsymbol{x}_{\mathrm{i}}, t_{\mathrm{i}}\right)=\frac{1}{\left\langle\psi_{\mathrm{f}} \mid \psi_{\mathrm{i}}\right\rangle} \bar{\psi}\left(\boldsymbol{x}, t \mid \boldsymbol{x}_{\mathrm{f}}, t_{\mathrm{f}}\right) \psi\left(\boldsymbol{x}, t \mid \boldsymbol{x}_{\mathrm{i}}, t_{\mathrm{i}}\right),
$$

where the associated wave function denoted by $\bar{\psi}$ is related to the so-called posterior conditional probability, $\bar{P}\left(\boldsymbol{x}, t \mid \boldsymbol{x}_{\mathrm{f}}, t_{\mathrm{f}}\right)$ for $t \leq t_{\mathrm{f}}$, by

$$
\bar{\psi}\left(\boldsymbol{x}, t \mid \boldsymbol{x}_{\mathrm{f}}, t_{\mathrm{f}}\right)=\phi_{0}(\boldsymbol{x})^{-1} \bar{P}\left(\boldsymbol{x}, t \mid \boldsymbol{x}_{\mathrm{f}}, t_{\mathrm{f}}\right),
$$

and obeys the backward wave equation,

$$
\frac{\partial}{\partial t} \bar{\psi}(\boldsymbol{x}, t)=\sum_{\boldsymbol{x}^{\prime}} H^{\dagger}\left(\boldsymbol{x}, \boldsymbol{x}^{\prime}\right) \bar{\psi}\left(\boldsymbol{x}^{\prime}, t\right) .
$$

Here $H^{\dagger}$ is the hermite conjugate of $H$, i.e. the transposed matrix in the present case. The eigensystem is common with the forward equation Eq.(9), when $H$ is hermitian, i.e. real and symmetric as has been assumed here.

The denominator in Eq.(15) is the weight of overlap between the two wave functions defined by an inner product,

$$
\left\langle\psi_{\mathrm{f}} \mid \psi_{\mathrm{i}}\right\rangle=\sum_{\boldsymbol{x}} \bar{\psi}\left(\boldsymbol{x}, t \mid \boldsymbol{x}_{\mathrm{f}}, t_{\mathrm{f}}\right) \psi\left(\boldsymbol{x}, t \mid \boldsymbol{x}_{\mathrm{i}}, t_{\mathrm{i}}\right) .
$$

Of course this quantity is real, while the corresponding quantity in the quantum mechanics is complex in general.

Let us define the ket- and the bra-vectors by

$$
\left|\psi_{\mathrm{i}}(t)\right\rangle=\left\{\psi\left(\boldsymbol{x}, t \mid \boldsymbol{x}_{\mathrm{i}}, t_{\mathrm{i}}\right)\right\}^{T} \text { and }\left\langle\psi_{\mathrm{f}}(t)\right|=\left\{\bar{\psi}\left(\boldsymbol{x}, t \mid \boldsymbol{x}_{\mathrm{f}}, t_{\mathrm{f}}\right)\right\} .
$$

Then the wave equations Eqs.(9) and (17) by assuming $H^{\dagger}=H$ are rewritten in the quantum mechanical form as

$$
\frac{\partial}{\partial t}\left|\psi_{\mathrm{i}}(t)\right\rangle=-H\left|\psi_{\mathrm{i}}(t)\right\rangle \text { and } \frac{\partial}{\partial t}\left\langle\psi_{\mathrm{f}}(t)\right|=\left\langle\psi_{\mathrm{f}}(t)\right| H,
$$

respectively. Henceforth, $H$ is called the Hamitonian.

where the Markovness, i.e. $P(f \mid x \cap i)=P(f \mid x)$ is assumed for the time order $t_{\mathrm{f}} \geq t \geq t_{\mathrm{i}}$. By applying the identity $(*)$ to $P(f \mid x)$ again, we obtain a symmetric expression,

$$
P(x \mid f \cap i)=\frac{P(x \mid f) P(f)}{P(x)} \frac{P(x \mid i) P(i)}{P(f \cap i)}=\frac{1}{R(f, i)} \frac{P(x \mid f) P(x \mid i)}{P(x)},
$$

where the first denominator $R(f, i)$ is given by

$$
R(f, i)=\frac{P(f \cap i)}{P(f) P(i)}=\sum_{x} \frac{P(x \mid f) P(x \mid i)}{P(x)},
$$

because of the normalization condition, $\sum_{x} P(x \mid f \cap i)=1 \forall f \cap i$. 
By using this pair of the Schrödinger equations it is shown that the overlap integral, or the inner product $\left\langle\psi_{\mathrm{f}} \mid \psi_{\mathrm{i}}\right\rangle$ given by Eq.(18) does not depend on the current time $t$, i.e.

$$
\frac{\partial}{\partial t}\left\langle\psi_{\mathrm{f}} \mid \psi_{\mathrm{i}}\right\rangle=\left\langle\psi_{\mathrm{f}}(t)|H| \psi_{\mathrm{i}}(t)\right\rangle-\left\langle\psi_{\mathrm{f}}(t)|H| \psi_{\mathrm{i}}(t)\right\rangle=0 .
$$

It should be noted that the present wave function $\psi$ satisfies a conservation law only in this meaning Eq.(18) coupled with its adjoint $\bar{\psi}$. In addition, it can be shown that this overlap integral has the following properties in the respective limits;

$$
\begin{aligned}
& \text { (i) } \lim _{t_{\mathrm{f}}-t_{\mathrm{i}} \rightarrow \infty}\left\langle\psi_{\mathrm{f}} \mid \psi_{\mathrm{i}}\right\rangle=1, \\
& \text { (ii) } \lim _{t_{\mathrm{f}}-t_{\mathrm{i}} \rightarrow 0}\left\langle\psi_{\mathrm{f}} \mid \psi_{\mathrm{i}}\right\rangle=\left[\phi_{0}\left(\boldsymbol{x}_{\mathrm{f}}\right) \phi_{0}\left(\boldsymbol{x}_{\mathrm{i}}\right)\right]^{-1} \delta\left(\boldsymbol{x}_{\mathrm{f}}-\boldsymbol{x}_{\mathrm{i}}\right) .
\end{aligned}
$$

Note that the TTCE (two-time conditional expectation) of a physical quantity $Q$ with respect to TTCP defined by

$$
\langle Q\rangle_{(\mathrm{f} ; \mathrm{i})}^{\mathrm{w}}=\sum_{\boldsymbol{x}} Q(\boldsymbol{x}) P\left(\boldsymbol{x}, t \mid \boldsymbol{x}_{\mathrm{f}}, t_{\mathrm{f}} ; \boldsymbol{x}_{\mathrm{i}}, t_{\mathrm{i}}\right)=\frac{\left\langle\psi_{\mathrm{f}}(t)|Q| \psi_{\mathrm{i}}(t)\right\rangle}{\left\langle\psi_{\mathrm{f}} \mid \psi_{\mathrm{i}}\right\rangle},
$$

has just the analogous form of the weak value in the quantum mechanics.

Thus the TTCP is a nonlinear quantity composed of a product of a pair of the forward and the backward wave functions, and cannot be described by a closed, linear evolution equation. Then it happens that the principle of the probability superposition is violated and the interference of wave functions may occur. However, its example is omitted here because none of remarkable phenomena from this view point has been found, yet. The reason may be that the wave functions are always real and positive in the present case. Therefore, let us discuss only the weak value in the rest.

\section{Stochastic model of classical Ising spins}

An example is a pair of the classical Ising spin $\sigma= \pm 1$ having an exchange interaction,

$$
E(\boldsymbol{x})=-J \sigma_{1} \sigma_{2},
$$

where $\boldsymbol{x}=\left(\sigma_{1}, \sigma_{2}\right)$. Let us number the stochastic variable $\boldsymbol{x}$ in the order, $(1,1),(1,-1),(-1,1),(-1,-1)$ and choose the following transition matrices,

$$
W=\left(\begin{array}{cccc}
0 & 1 & 1 & 0 \\
p^{2} & 0 & 0 & p^{2} \\
p^{2} & 0 & 0 & p^{2} \\
0 & 1 & 1 & 0
\end{array}\right) \quad \text { or } \quad L=\left(\begin{array}{cccc}
2 p^{2} & -1 & -1 & 0 \\
-p^{2} & 2 & 0 & -p^{2} \\
-p^{2} & 0 & 2 & -p^{2} \\
0 & -1 & -1 & 2 p^{2}
\end{array}\right)
$$


where $p=\mathrm{e}^{-\beta J}, \beta=1 / k_{\mathrm{B}} T$. Evidently this transition matrix $W$ satisfies the detailed balance condition,

$$
\mathrm{e}^{-\beta E(\boldsymbol{x})} W\left(\boldsymbol{x} \rightarrow \boldsymbol{x}^{\prime}\right)=\mathrm{e}^{-\beta E\left(\boldsymbol{x}^{\prime}\right)} W\left(\boldsymbol{x}^{\prime} \rightarrow \boldsymbol{x}\right),
$$

at the steady state, i.e. the thermal equilibrium of a temperature $T$. With the use of the equilibrium distribution function,

$$
P_{0}(\boldsymbol{x})=\frac{1}{2\left(1+p^{2}\right)}\left(1, p^{2}, p^{2}, 1\right) \text { and } \phi_{0}(\boldsymbol{x})=\frac{1}{\sqrt{2\left(1+p^{2}\right)}}(1, p, p, 1),
$$

we find the corresponding hermitian Hamiltonian,

$$
\begin{aligned}
H & =\left(\begin{array}{cccc}
2 p^{2} & -p & -p & 0 \\
-p & 2 & 0 & -p \\
-p & 0 & 2 & -p \\
0 & -p & -p & 2 p^{2}
\end{array}\right) \\
& =\left(1+p^{2}\right) \sigma_{0} \otimes \sigma_{0}-\left(1-p^{2}\right) \sigma_{z} \otimes \sigma_{z}-p\left(\sigma_{0} \otimes \sigma_{x}+\sigma_{x} \otimes \sigma_{0}\right),
\end{aligned}
$$

where $\sigma_{x}$ and $\sigma_{z}$ are the usual Pauli matrices and $\sigma_{0}$ denotes the two dimensional unit matrix $I_{2}$. This is the Hamiltonian of a pair of quantum Ising spins with an exchange interaction in a transverse magnetic field.

The eigenvalues and the eigenstates of this Hamiltonian $H$,

$$
\left\{\begin{array}{l}
\lambda_{0}=0, \lambda_{1}=2 p^{2}, \lambda_{2}=2, \lambda_{3}=2\left(1+p^{2}\right), \\
|0\rangle=\frac{1}{\sqrt{2\left(1+p^{2}\right)}}[|\uparrow \uparrow\rangle+p|\uparrow \downarrow\rangle+p|\downarrow \uparrow\rangle+|\downarrow \downarrow\rangle], \\
|1\rangle=\frac{1}{\sqrt{2}}[|\uparrow \uparrow\rangle-|\downarrow \downarrow\rangle], \\
|2\rangle=\frac{1}{\sqrt{2}}[|\uparrow \downarrow\rangle-|\downarrow \uparrow\rangle], \\
|3\rangle=\frac{1}{\sqrt{2\left(1+p^{2}\right)}}[p|\uparrow \uparrow\rangle-|\uparrow \downarrow\rangle-|\downarrow \uparrow\rangle+p|\downarrow \downarrow\rangle],
\end{array}\right.
$$

can be easily obtained, where $|0\rangle=\left|\phi_{0}\right\rangle$, the ground state. Here the familiar notations $\uparrow, \downarrow$ are used for $\sigma= \pm 1$. Note that the first excited state is almost degenerate with the ground state for a small transition probability $p^{2}$.

By using this eigensystem we can calculate the state vectors, $\left|\psi_{\mathrm{i}}(t)\right\rangle$ and $\left\langle\psi_{\mathrm{f}}(t)\right|$ for arbitrary initial and final states in just the same manner of the elementary quantum mechanics except for the fact that the time $t$ is imaginary.

Strange behaviors can be expected only when the paths from $i$ to $f$ are very rare cases, because the post-selection causes little effect when the 


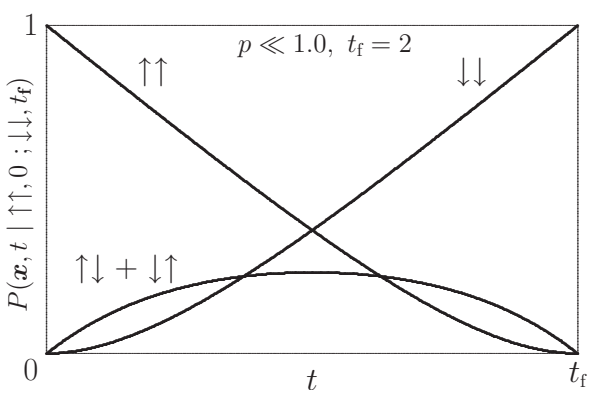

Fig. 2. Two-time conditional probability

paths are dominant ones. Then let us consider the case where the initial and the final states differ from each other. Let

$$
\boldsymbol{x}_{\mathrm{i}}=\uparrow \uparrow \text { at } t=0 \text { and } \boldsymbol{x}_{\mathrm{f}}=\downarrow \downarrow \text { at } t=t_{\mathrm{f}},
$$

that is,

$$
P(\boldsymbol{x}, 0)=(1,0,0,0) \text { and } \bar{P}\left(\boldsymbol{x}, t_{\mathrm{f}}\right)=(0,0,0,1),
$$

or equivalently,

$$
\left|\psi_{\mathrm{i}}(0)\right\rangle=\sqrt{2\left(1+p^{2}\right)}|\uparrow \uparrow\rangle \text { and }\left\langle\psi_{\mathrm{f}}\left(t_{\mathrm{f}}\right)\right|=\sqrt{2\left(1+p^{2}\right)}\langle\downarrow \downarrow| .
$$

By using the eigenvector expansion we obtain,

$$
\begin{aligned}
& \left|\psi_{\mathrm{i}}(t)\right\rangle=|0\rangle+\sqrt{1+p^{2}} \mathrm{e}^{-\lambda_{1} t}|1\rangle+p \mathrm{e}^{-\lambda_{3} t}|3\rangle, \\
& \left\langle\psi_{\mathrm{f}}(t)\right|=\langle 0|-\sqrt{1+p^{2}} \mathrm{e}^{-\lambda_{1}\left(t_{\mathrm{f}}-t\right)}\langle 1|+p \mathrm{e}^{-\lambda_{3}\left(t_{\mathrm{f}}-t\right)}\langle 3|,
\end{aligned}
$$

and

$$
\left\langle\psi_{\mathrm{f}} \mid \psi_{\mathrm{i}}\right\rangle=1-\left(1+p^{2}\right) \mathrm{e}^{-\lambda_{1} t_{\mathrm{f}}}+p^{2} \mathrm{e}^{-\lambda_{3} t_{\mathrm{f}}}(>0) .
$$

The TTCP is shown in Figure.2. This result itself is very natural and well-expected, all probabilities being always non-negative.

A strange behavior appears when we use the basis $\{|k\rangle, k=0,1,2,3\}$, the eigenstates of the Hamiltonian $H$ instead of the spin states $\{|\boldsymbol{x}\rangle=$ $\left.\left|\sigma_{1} \sigma_{2}\right\rangle\right\}$. We can calculate the virtual probability, i.e. the TTCE of the projection operator $|k\rangle\langle k|$ onto each eigenstate $|k\rangle$ in the same manner. The result is given by 


$$
\begin{aligned}
& P(0, t)=\frac{\left\langle\psi_{\mathrm{f}}(t) \mid 0\right\rangle\left\langle 0 \mid \psi_{\mathrm{i}}(t)\right\rangle}{\left\langle\psi_{\mathrm{f}} \mid \psi_{\mathrm{i}}\right\rangle}=\frac{1}{\left\langle\psi_{\mathrm{f}} \mid \psi_{\mathrm{i}}\right\rangle}, \\
& P(1, t)=\frac{\left\langle\psi_{\mathrm{f}}(t) \mid 1\right\rangle\left\langle 1 \mid \psi_{\mathrm{i}}(t)\right\rangle}{\left\langle\psi_{\mathrm{f}} \mid \psi_{\mathrm{i}}\right\rangle}=-\frac{\left(1+p^{2}\right) \mathrm{e}^{-\lambda_{1} t_{\mathrm{f}}}}{\left\langle\psi_{\mathrm{f}} \mid \psi_{\mathrm{i}}\right\rangle}(<0), \\
& P(2, t)=\frac{\left\langle\psi_{\mathrm{f}}(t) \mid 2\right\rangle\left\langle 2 \mid \psi_{\mathrm{i}}(t)\right\rangle}{\left\langle\psi_{\mathrm{f}} \mid \psi_{\mathrm{i}}\right\rangle}=0, \\
& P(3, t)=\frac{\left\langle\psi_{\mathrm{f}}(t) \mid 3\right\rangle\left\langle 3 \mid \psi_{\mathrm{i}}(t)\right\rangle}{\left\langle\psi_{\mathrm{f}} \mid \psi_{\mathrm{i}}\right\rangle}=\frac{p^{2} \mathrm{e}^{-\lambda_{3} t_{\mathrm{f}}}}{\left\langle\psi_{\mathrm{f}} \mid \psi_{\mathrm{i}}\right\rangle} .
\end{aligned}
$$

The fictitious negative probability is found in $P(1, t)$. Of course the completeness of the probability, $\sum_{k=0}^{3} P(k, t)=1$, is satisfied evidently because of Eq.(27).

This negativity is precisely expected from the signs of the expansion coefficients of the eigenvectors in the right side of Eq.(25). Some of inner products $\langle\boldsymbol{x} \mid k\rangle$ between two basis systems $\left\{|\boldsymbol{x}\rangle=\left|\sigma_{1} \sigma_{2}\right\rangle\right\}$ and $\{|k\rangle\}$ are found to be negative. Then some part of the virtual TTCP happens to be negative, when the initial and the final states differ from each other.

On the contrary, when the both states are the same, this situation cannot be expected, because the negative inner products, if any, would be squared. For example, when we select as

$$
\boldsymbol{x}_{\mathrm{i}}=\boldsymbol{x}_{\mathrm{f}}=\uparrow \uparrow \text { at } t=0 \text { and } t=t_{\mathrm{f}},
$$

we find the corresponding virtual probabilities all positive, i.e. ${ }^{\mathrm{d}}$

$$
\begin{aligned}
& P(0, t)=\frac{\left\langle\psi_{\mathrm{i}}(t) \mid 0\right\rangle\left\langle 0 \mid \psi_{\mathrm{i}}(t)\right\rangle}{\left\langle\psi_{\mathrm{i}} \mid \psi_{\mathrm{i}}\right\rangle}=\frac{1}{\left\langle\psi_{\mathrm{i}} \mid \psi_{\mathrm{i}}\right\rangle}, \\
& P(1, t)=\frac{\left\langle\psi_{\mathrm{i}}(t) \mid 1\right\rangle\left\langle 1 \mid \psi_{\mathrm{i}}(t)\right\rangle}{\left\langle\psi_{\mathrm{i}} \mid \psi_{\mathrm{i}}\right\rangle}=\frac{\left(1+p^{2}\right) \mathrm{e}^{-\lambda_{1} t_{\mathrm{f}}}}{\left\langle\psi_{\mathrm{i}} \mid \psi_{\mathrm{i}}\right\rangle}, \\
& P(2, t)=\frac{\left\langle\psi_{\mathrm{i}}(t) \mid 2\right\rangle\left\langle 2 \mid \psi_{\mathrm{i}}(t)\right\rangle}{\left\langle\psi_{\mathrm{i}} \mid \psi_{\mathrm{i}}\right\rangle}=0, \\
& P(3, t)=\frac{\left\langle\psi_{\mathrm{i}}(t) \mid 3\right\rangle\left\langle 3 \mid \psi_{\mathrm{i}}(t)\right\rangle}{\left\langle\psi_{\mathrm{i}} \mid \psi_{\mathrm{i}}\right\rangle}=\frac{p^{2} \mathrm{e}^{-\lambda_{3} t_{\mathrm{f}}}}{\left\langle\psi_{\mathrm{i}} \mid \psi_{\mathrm{i}}\right\rangle},
\end{aligned}
$$

where

$$
\left\langle\psi_{\mathrm{i}} \mid \psi_{\mathrm{i}}\right\rangle=1+\left(1+p^{2}\right) \mathrm{e}^{-\lambda_{1} t_{\mathrm{f}}}+p^{2} \mathrm{e}^{-\lambda_{3} t_{\mathrm{f}}}
$$

${ }^{\mathrm{d}}$ Note that the bra- and the ket-vectors in these expressions denote

$$
\left|\psi_{\mathrm{i}}(t)\right\rangle=\mathrm{e}^{-t H}\left|\psi_{\mathrm{i}}\right\rangle \text { and }\left\langle\psi_{\mathrm{i}}(t)\right|=\left\langle\psi_{\mathrm{i}}\right| \mathrm{e}^{-\left(t_{\mathrm{f}}-t\right) H},
$$

from the present definitions Eq.(19) of them, and the overlap integral $\left\langle\psi_{\mathrm{i}} \mid \psi_{\mathrm{i}}\right\rangle$ in the denominators is better to be written explicitly as $\left\langle\psi_{\mathrm{i}}(t) \mid \psi_{\mathrm{i}}(t)\right\rangle$, or $\left\langle\psi_{\mathrm{i}}\left|\mathrm{e}^{-t_{\mathrm{f}} H}\right| \psi_{\mathrm{i}}\right\rangle$ to avoid a confusion, as if $\left\langle\psi_{\mathrm{i}} \mid \psi_{\mathrm{i}}\right\rangle=1$ in the usual quantum mechanical notation. 


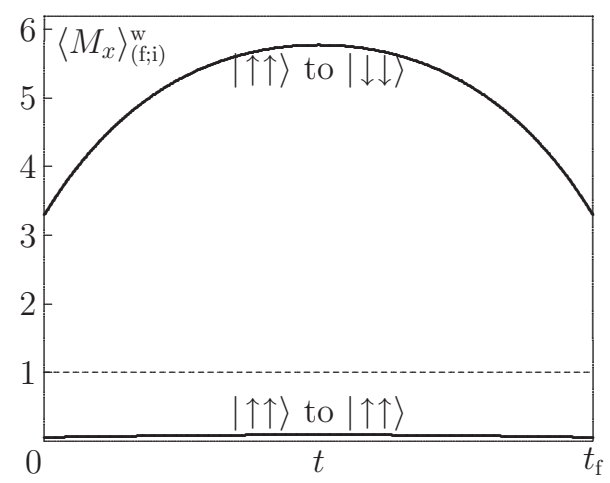

Fig. 3. Abnormal and normal TTCE of the transverse magnetization $M_{x}$ for the transition probability $p=0.2$ and $p^{2} t_{\mathrm{f}}=0.01$. The indicators $|\uparrow \uparrow\rangle$ and $|\downarrow \downarrow\rangle$ denote the initial and the final states of the respective TTCP.

This is a general conclusion for two different bases $\left\{\boldsymbol{e}_{i}\right\}$ and $\left\{\boldsymbol{e}_{j}^{\prime}\right\}$ of any real vector space, because at least one of the inner products, $\left\{\boldsymbol{e}_{i} \cdot \boldsymbol{e}_{j}^{\prime}\right\}$ must be negative.

That is, the negative probability can be expected at least when

(1) the initial and the final states differ from each other,

(2) and the orthogonal basis of the intermediate projection differs from the basis of the initial and the final selections, so that one of the inner products is negative.

The same situation may occur in the quantum system between different sets of eigenvectors of non-commutative observables, say, $P, Q$. When we select the initial and the final states as different eigenstates of $P$, the virtual TTCP, i.e. the weak value of the projection operator $|q\rangle\langle q|$ onto some of the eigenstates of $Q$ can be negative. This setting is sufficient for the condition ${ }^{5}$ to find the strange weak value.

A strange behavior related to this negative probability is the abnormal enhancement of some observables as is stated in Sec.1. An example is shown in Fig. 3 for a quantity, say, the transverse magnetization,

$$
M_{x}=\frac{1}{2}\left(\sigma_{x} \otimes \sigma_{0}+\sigma_{0} \otimes \sigma_{x}\right) .
$$




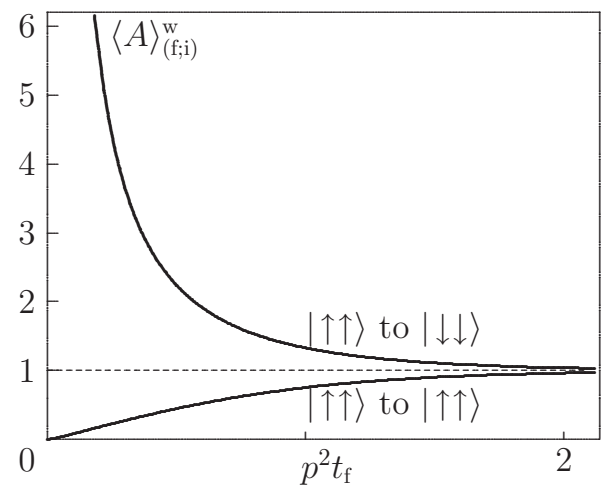

Fig. 4. Abnormal and normal TTCE of $A=\sigma_{x} \otimes \sigma_{x}$ for $p=0.2$.

An abnormal behavior

$$
\begin{aligned}
\left\langle M_{x}\right\rangle_{(\mathrm{f} ; \mathrm{i})}^{\mathrm{w}} & =\frac{1}{\left\langle\psi_{\mathrm{f}} \mid \psi_{\mathrm{i}}\right\rangle}\left[\frac{2 p}{1+p^{2}}\left(1-p^{2} \mathrm{e}^{-\lambda_{3} t_{\mathrm{f}}}\right)-\frac{1-p^{2}}{1+p^{2}}\left(\mathrm{e}^{-\lambda_{3} t}+\mathrm{e}^{-\lambda_{3}\left(t_{\mathrm{f}}-t\right)}\right)\right] \\
& >1
\end{aligned}
$$

is found for sufficiently small $p$ and $t_{\mathrm{f}}$. Note that the natural norm of $M_{x}$ must be less than 1 , because the eigenvalue spectrum of $M_{x}$ is $\{-1,0,0,1\}$. When the transition rate is very small, i.e. $p^{2} t_{\mathrm{f}} \ll 1$, we find

$$
\left\langle M_{x}\right\rangle_{(\mathrm{f} ; \mathrm{i})}^{\mathrm{w}} \gg 1
$$

A plain reason of this singular behavior is that the overlap integral $\left\langle\psi_{\mathrm{f}} \mid \psi_{\mathrm{i}}\right\rangle$ in the denominator may be expected to be very small owing to (ii) of Eq.(21), whenever the initial and the final states differ from each other, i.e. $\boldsymbol{x}_{\mathrm{i}} \neq \boldsymbol{x}_{\mathrm{f}}$. This means that to reach $\boldsymbol{x}_{\mathrm{f}}=(\downarrow \downarrow)$ starting from $\boldsymbol{x}_{\mathrm{i}}=(\uparrow \uparrow)$ in a given time occurs scarcely and is far from the main flow of the conditional probability. On the contrary none of such strange behaviors are found when $\boldsymbol{x}_{\mathrm{i}}=\boldsymbol{x}_{\mathrm{f}}$, e.g. $x_{\mathrm{i}}=\boldsymbol{x}_{\mathrm{f}}=(\uparrow \uparrow)$. The result for the latter case for the same parameters as the upper abnormal case is shown by the lower curve in Fig.3, its maximum being $\sim 0.09$ at $t=t_{\mathrm{f}} / 2$ and minimum $\sim 0.05$ at $t=0$ and $t_{\mathrm{f}}$.

In Fig. 4 the TTCE of another quantity $A=\sigma_{x} \otimes \sigma_{x}$ having a spectrum $\{-1,-1,1,1\}$ are shown also. Note that $A$ is commutative with $H$ and is a conserved quantity. Then the horizontal axis in this figure shows a parameter of the transition probability instead of the current time itself. 
(Single spin model) The previous model having an energy barrier is aimed at realizing the rare paths from $i$ to $f$ in order to find out a strange weak value easily. After the symposium, however, the author noticed that a single, free Ising spin system provides a simpler example.

An Ising spin flips up-to-down and down-to-up randomly. A transition probability may be defined by

$$
W=\left(\begin{array}{ll}
0 & p \\
p & 0
\end{array}\right) \text { and } L=\left(\begin{array}{cc}
p & -p \\
-p & p
\end{array}\right),
$$

whose equilibrium state is

$$
P_{0}(\boldsymbol{x})=\left(\frac{1}{2}, \frac{1}{2}\right) \quad \text { or } \quad \phi_{0}(\boldsymbol{x})=\left(\frac{1}{\sqrt{2}}, \frac{1}{\sqrt{2}}\right) .
$$

Then, the Hamiltonian for this classical stochastic model is given by $\mathrm{e}$

$$
H=\left(\begin{array}{cc}
p & -p \\
-p & p
\end{array}\right)=p\left(\sigma_{0}-\sigma_{x}\right) .
$$

Another eigenstate, i.e. the excited state is

$$
\lambda_{1}=2 p \text { and } \phi_{1}(\boldsymbol{x})=\left(\frac{1}{\sqrt{2}},-\frac{1}{\sqrt{2}}\right),
$$

that is, the two eigenstates of $H$ are those of $\sigma_{x}$ itself, i.e. the Hadamard states,

$$
|0\rangle=\frac{1}{\sqrt{2}}(|\uparrow\rangle+|\downarrow\rangle) \text { and }|1\rangle=\frac{1}{\sqrt{2}}(|\uparrow\rangle-|\downarrow\rangle) .
$$

Let us select the initial and the final states as

$$
\boldsymbol{x}_{\mathrm{i}}=\uparrow \quad \text { at } t=0, \quad \boldsymbol{x}_{\mathrm{f}}=\downarrow \quad \text { at } t=t_{\mathrm{f}} .
$$

Then we find

$$
\left|\psi_{\mathrm{i}}(t)\right\rangle=|0\rangle+\mathrm{e}^{-2 p t}|1\rangle, \quad\left\langle\psi_{\mathrm{f}}(t)\right|=\langle 0|-\mathrm{e}^{-2 p\left(t_{\mathrm{f}}-t\right)}\langle 1|,
$$

and

$$
\left\langle\psi_{\mathrm{f}} \mid \psi_{\mathrm{i}}\right\rangle=1-\mathrm{e}^{-2 p t_{\mathrm{f}}},
$$

${ }^{\mathrm{e}} \mathrm{A}$ quantum free spin system has been used often to demonstrate the weak value in quantum mechanics, but it differs from the present system which has a transverse magnetic field $p$ as shown by this Hamiltonian. 
by using the eigenvector expansion, where the negative expansion coefficient appears. After the same procedures as the previous model we obtain an extraordinary TTCP,

$$
\begin{aligned}
& P(0, t)=\frac{\left\langle\psi_{\mathrm{f}}(t) \mid 0\right\rangle\left\langle 0 \mid \psi_{\mathrm{i}}(t)\right\rangle}{\left\langle\psi_{\mathrm{f}} \mid \psi_{\mathrm{i}}\right\rangle}=\frac{1}{1-\mathrm{e}^{-2 p t_{\mathrm{f}}}}>1, \\
& P(1, t)=\frac{\left\langle\psi_{\mathrm{f}}(t) \mid 1\right\rangle\left\langle 1 \mid \psi_{\mathrm{i}}(t)\right\rangle}{\left\langle\psi_{\mathrm{f}} \mid \psi_{\mathrm{i}}\right\rangle}=-\frac{\mathrm{e}^{-2 p t_{\mathrm{f}}}}{1-\mathrm{e}^{-2 p t_{\mathrm{f}}}}<0,
\end{aligned}
$$

and a strange weak value,

$$
\left\langle\sigma_{x}\right\rangle_{(\mathrm{f} ; \mathrm{i})}^{\mathrm{w}}=\operatorname{coth} p t_{\mathrm{f}}>1
$$

again.

On the contrary, when $\boldsymbol{x}_{\mathrm{i}}=\boldsymbol{x}_{\mathrm{f}}=\uparrow$, we find an ordinary TTCP,

$$
\begin{aligned}
& P(0, t)=\frac{1}{1+\mathrm{e}^{-2 p t_{\mathrm{f}}}}>0, \\
& P(1, t)=\frac{\mathrm{e}^{-2 p t_{\mathrm{f}}}}{1+\mathrm{e}^{-2 p t_{\mathrm{f}}}}>0,
\end{aligned}
$$

and

$$
\left\langle\sigma_{x}\right\rangle_{(\mathrm{f} ; \mathrm{i})}^{\mathrm{w}}=\tanh p t_{\mathrm{f}}<1 .
$$

Thus the origin of the negative probability and the strange weak value is more obvious in this simplest system.

\section{Extension of TTCP to a density matrix}

It should be noted that the physical quantities $M_{x}$ and $A$ in the previous section are non-diagonal in the spin-state representation and have no corresponding quantities in the classical Ising spin system. They are related to the transition rate of the stochastic Ising spin. In order to calculate the expectations of such non-diagonal quantities we need an extension of the TTCP to the two-time conditional density matrix defined by

$$
\begin{aligned}
\rho_{(\mathrm{f} ; \mathrm{i})}^{\mathrm{w}}(t) & =\frac{1}{\left\langle\psi_{\mathrm{f}} \mid \psi_{\mathrm{i}}\right\rangle}\left|\psi_{\mathrm{i}}(t)\right\rangle\left\langle\psi_{\mathrm{f}}(t)\right| \\
& =\frac{1}{\left\langle\psi_{\mathrm{f}} \mid \psi_{\mathrm{i}}\right\rangle} \sum_{\boldsymbol{x}, \boldsymbol{x}^{\prime}} \bar{\psi}\left(\boldsymbol{x}^{\prime}, t \mid \boldsymbol{x}_{\mathrm{f}}, t_{\mathrm{f}}\right) \psi\left(\boldsymbol{x}, t \mid \boldsymbol{x}_{\mathrm{i}}, 0\right)|\boldsymbol{x}\rangle\left\langle\boldsymbol{x}^{\prime}\right| .
\end{aligned}
$$

From the definition Eq.(18) of the overlap integral $\left\langle\psi_{\mathrm{f}} \mid \psi_{\mathrm{i}}\right\rangle$, it is evident that

$$
\operatorname{Tr} \rho_{(\mathrm{f} ; \mathrm{i})}^{\mathrm{w}}(t)=\frac{1}{\left\langle\psi_{\mathrm{f}} \mid \psi_{\mathrm{i}}\right\rangle} \sum_{\boldsymbol{x}} \bar{\psi}\left(\boldsymbol{x}, t \mid \boldsymbol{x}_{\mathrm{f}}, t_{\mathrm{f}}\right) \psi\left(\boldsymbol{x}, t \mid \boldsymbol{x}_{\mathrm{i}}, 0\right)=1
$$


It should be noted, however, that the diagonal elements of this density matrix are not always positive as is shown by Eq.(28) in Sec.4, when it is diagonalized by using the basis $\{|k\rangle, k=0,1,2,3\}$, the eigenstates of the Hamiltonian $H$.

With the use of this density matrix the definition Eq.(22) of the TTCE is extended as

$$
\langle Q\rangle_{(\mathrm{f} ; \mathrm{i})}^{\mathrm{w}}=\operatorname{Tr} \rho_{(\mathrm{f} ; \mathrm{i})}^{\mathrm{w}} Q .
$$

Of course this definition of the TTCE results in the classical one, if $Q$ is a diagonal quantity.

The notion of this density matrix has not been used in the conventional classical stochastic process. It should be emphasized, however, that this quantity is within a scheme of the classical stochastic process itself, because the wave functions, $\psi$ and $\bar{\psi}$ in Eq.(40) are related to the forward and the posterior, classical conditional probabilities, respectively. In addition, we have an alternative expression for $\bar{\psi}$,

$$
\bar{\psi}\left(\boldsymbol{x}^{\prime}, t \mid \boldsymbol{x}_{\mathrm{f}}, t_{\mathrm{f}}\right)=\psi\left(\boldsymbol{x}^{\prime}, t_{\mathrm{f}} \mid \boldsymbol{x}_{\mathrm{f}}, t\right) \quad\left(=\phi_{0}\left(\boldsymbol{x}^{\prime}\right)^{-1} P\left(\boldsymbol{x}^{\prime}, t_{\mathrm{f}}-t \mid \boldsymbol{x}_{\mathrm{f}}, 0\right)\right),
$$

or equivalently,

$$
\begin{aligned}
\bar{P}\left(\boldsymbol{x}^{\prime}, t \mid \boldsymbol{x}_{\mathrm{f}}, t_{\mathrm{f}}\right) P_{0}\left(\boldsymbol{x}_{\mathrm{f}}\right) & =P\left(\boldsymbol{x}_{\mathrm{f}}, t_{\mathrm{f}} \mid \boldsymbol{x}^{\prime}, t\right) P_{0}\left(\boldsymbol{x}^{\prime}\right) \\
& =P\left(\boldsymbol{x}^{\prime}, t_{\mathrm{f}} \mid \boldsymbol{x}_{\mathrm{f}}, t\right) P_{0}\left(\boldsymbol{x}_{\mathrm{f}}\right),
\end{aligned}
$$

for $t \leq t_{\mathrm{f}}$ due to the time-reversal symmetry corresponding to the detailed balance. Then the density matrix Eq.(40) can be written as

$$
\rho_{(\mathrm{f} ; \mathrm{i})}^{\mathrm{w}}(t)=\frac{1}{\left\langle\psi_{\mathrm{f}} \mid \psi_{\mathrm{i}}\right\rangle} \sum_{\boldsymbol{x}, \boldsymbol{x}^{\prime}} \frac{P\left(\boldsymbol{x}^{\prime}, t_{\mathrm{f}}-t \mid \boldsymbol{x}_{\mathrm{f}}, 0\right) P\left(\boldsymbol{x}, t \mid \boldsymbol{x}_{\mathrm{i}}, 0\right)}{\phi_{0}\left(\boldsymbol{x}^{\prime}\right) \phi_{0}(\boldsymbol{x})}|\boldsymbol{x}\rangle\left\langle\boldsymbol{x}^{\prime}\right|,
$$

while the overlap integral can be re-defined by

$$
\left\langle\psi_{\mathrm{f}} \mid \psi_{\mathrm{i}}\right\rangle=\sum_{\boldsymbol{x}} \frac{P\left(\boldsymbol{x}, t_{\mathrm{f}}-t \mid \boldsymbol{x}_{\mathrm{f}}, 0\right) P\left(\boldsymbol{x}, t \mid \boldsymbol{x}_{\mathrm{i}}, 0\right)}{P_{0}(\boldsymbol{x})} .
$$

This fact means that we can define the TTCP and the corresponding density matrix with only a pair of the usual, forward conditional probabilities for two individual initial states, $\boldsymbol{x}_{\mathrm{i}}$ and $\boldsymbol{x}_{\mathrm{f}}$. We need no data discarding due to the post-selection.

\section{Summary and discussions}

Except for the facts that the time is imaginary and the wave function is always real and positive, the classical stochastic process can be described 
in an analogous form of the quantum mechanics, if we use the TTCP. For example, the abnormal behaviors of the weak value in the quantum mechanics are emulated. The TTCP and its TTCE, i.e. the weak values are always real in the present classical case. Therefore, the origin of such abnormal behaviors is clearer than the quantum mechanical case where complex quantities appear.

In addition, if we have not the explicit solution of the eigenvalue problem, we may calculate the weak value at least with use of a Monte-Carlo simulation which is often used to investigate the stochastic model. In performing a simulation it should be noted that we can calculate the TTCP and its TTCE with two usual, forward conditional probabilities for respective initial conditions, the pre-selected and the post-selected ones, when the detailed balance condition is satisfied.

The importance of the weak value in the quantum mechanics is that it is related to the new notion of the weak measurement without disturbing the quantum state. An analogous notion of the latter in the classical stochastic process, if any, has not been found yet.

\section{Acknowledgment}

This work is supported by Open Research Center Project for Private Universities: Matching fund subsidy from MEXT of Japan.

\section{References}

1. Y. Aharonov, D. Z. Albert and L. Vaidman, Phys. Rev. Letters, 60, 1351, (1988).

2. N. W. M. Ritchie, J. G. Story and R. G. Hulet, Phys. Rev. Letters, 66, 1107, (1991).

3. A. Hosoya, A lecture note for ORC Summer School on Decoherence, Entanglement and Entropy held at Kobe, Japan, 8-11 Aug, 2009.

4. Y. Aharonov, P. G. Bergmann and J.L. Lebowitz, Phys. Rev. 134, B1410, (1964).

5. A. Hosoya and Y. Shikano, Jour. Phys. A: Math. Theor. 43, 385307, (2010). (Accurate and detailed references on the present topic are found therein.)

6. R. P. Feynman, 'Negative Probability' in Quantum Implications, Routledge \& Kegan Paul, London, (1987), p.235-248.

7. D. Sokolovski, Phys. Rev. A 76, 042125, (2007).

8. Y. Aharonov and A. Botero, Phys. Rev. A, 72, 052111, (2005).

9. For example, R. Kubo, K. Matsuo and K. Kitahara, Jour. Stat. Phys. 9, 51, (1973).

10. L. Onsager, Phys. Rev. 37, 405 (1931).

11. H. Tomita, A. Ito and H. Kidachi, Prog. Theor. Phys. 56, 786, (1976). 